KHATS' R.V.

\title{
REGULAR GROWTH OF FOURIER COEFFICIENTS OF THE LOGARITHMIC DERIVATIVE OF ENTIRE FUNCTIONS OF IMPROVED REGULAR GROWTH
}

\begin{abstract}
We establish a criterion for the improved regular growth of entire functions of positive order with zeros on a finite system of rays in terms of Fourier coefficients of their logarithmic derivative.

Key words and phrases: entire function of completely regular growth, entire function of improved regular growth, logarithmic derivative, Fourier coefficients, finite system of rays.
\end{abstract}

Drohobych Ivan Franko State Pedagogical University, Institute of Physics, Mathematics, Economy and Innovation Technologies, 3 Stryis'ka Str., 82100 Drohobych, Ukraine

e-mail: khats@ukr.net

\section{INTRODUCTION}

Let $f$ be an entire function, let $f(0)=1$, let $F(z):=z f^{\prime}(z) / f(z), z=r e^{i \varphi}$, let $\left(\lambda_{n}\right)$ be the sequence of its zeros, let $\Omega=\left\{\left|\lambda_{n}\right|: n \in \mathbb{N}\right\}$, let $p$ be the least nonnegative integer number for which $\sum_{n \in \mathbb{N}}\left|\lambda_{n}\right|^{-p-1}<+\infty$, let $n_{k}(r, f):=\sum_{\left|\lambda_{n}\right| \leq r} e^{-i k \arg \lambda_{n}}, k \in \mathbb{Z}$, let $n(r, \psi ; f):=$ $\sum_{\left|\lambda_{n}\right| \leq r, \arg \lambda_{n}=\psi} 1$, let $Q_{\rho}$ be the coefficient of $z^{\rho}$ in the exponential factor in the Hadamard-Borel representation $([12$, p. 24]) of an entire function $f$ of order $\rho \in(0,+\infty)$, and let

$$
\begin{aligned}
& c_{k}(r, \log |f|):=\frac{1}{2 \pi} \int_{0}^{2 \pi} e^{-i k \varphi} \log \left|f\left(r e^{i \varphi}\right)\right| d \varphi, \quad k \in \mathbb{Z}, \quad r>0, \\
& c_{k}(r, F):=\frac{1}{2 \pi} \int_{0}^{2 \pi} e^{-i k \varphi} F\left(r e^{i \varphi}\right) d \varphi, \quad k \in \mathbb{Z}, \quad r>0, \quad r \notin \Omega,
\end{aligned}
$$

be a Fourier coefficients of the functions $\log \left|f\left(r e^{i \varphi}\right)\right|$ and $F\left(r e^{i \varphi}\right)$, respectively. A set $C \subset \mathbb{C}$ is called a $C^{0}-$ set $([12$, p. 90$])$ if it can be covered by a system of disks $\left\{z:\left|z-a_{k}\right|<s_{k}\right\}$, $k \in \mathbb{N}$, satisfying $\sum_{\left|a_{k}\right| \leq r} s_{k}=o(r)$ as $r \rightarrow+\infty$. A set $E \subset[0,+\infty)$ is called a $E_{0}$-set $([12$, p. 96]) if $\operatorname{mes}(E \cap[0, r])=o(r)$ as $r \rightarrow+\infty$.

УДК 517.5

2010 Mathematics Subject Classification: 30D15, 30D20, 30D30.

(C) Khats' R.V., 2019 
An entire function $f$ of order $\rho \in(0,+\infty)$ with the indicator $h(\varphi)$ is called an entire function of completely regular growth in the sense of Levin and Pfluger ([12, p. 139]) if there exists a $C^{0}$-set such that

$$
\log \left|f\left(r e^{i \varphi}\right)\right|=r^{\rho} h(\varphi)+o\left(r^{\rho}\right), \quad C^{0} \not \supset r e^{i \varphi} \rightarrow \infty,
$$

uniformly in $\varphi \in[0,2 \pi)$. Numerous investigations have been devoted to the development of the Levin-Pfluger theory of entire functions and generalization of its results to other classes of functions (see $[1,3,11,12]$ ). At present, many different conditions are known that are necessary and sufficient for the completely regular growth of entire functions. In particular, from $[2,4,5]$ it follows a criterion for the completely regular growth of entire functions of positive order in terms of Fourier coefficients of their logarithmic derivative.

Theorem A $([2,4,5])$. For an entire function $f$ of order $\rho \in(0,+\infty)$ to be a function of completely regular growth, it is necessary and sufficient that for all $k \in \mathbb{Z}$

$$
c_{k}(r, F)=d_{k} r^{\rho}+o\left(r^{\rho}\right), \quad r \rightarrow+\infty, \quad r \notin E_{0}, \quad d_{k} \in \mathbb{C} .
$$

In $[7,15]$ (see also $[8,9,10,14]$ ), the notion of entire function of improved regular growth was introduced, and a criterion for this regularity was obtained in terms of the distribution of zeros under the condition that they are located on a finite system of rays. In [6], this notion was generalized to subharmonic functions. Criterion for the improved regular growth of entire functions of positive order with zeros on a finite system of rays in terms of their Fourier coefficients was established in [8]. Asymptotic behavior of entire functions of improved regular growth with zeros on a finite system of rays in the metric of $L^{p}[0,2 \pi]$ was described in [10].

An entire function $f$ is called a function of improved regular growth $([7,8,9,10,14,15])$ if for certain $\rho \in(0,+\infty)$ and $\rho_{1} \in(0, \rho)$, and a $2 \pi$-periodic $\rho$-trigonometrically convex function $h(\varphi) \not \equiv-\infty$ there exists a set $U \subset \mathbb{C}$ contained in the union of disks with finite sum of radii and such that

$$
\log |f(z)|=|z|^{\rho} h(\varphi)+o\left(|z|^{\rho_{1}}\right), \quad U \not \supset z=r e^{i \varphi} \rightarrow \infty .
$$

If an entire function $f$ is of improved regular growth, then it has the order $\rho$ and indicator $h(\varphi)([15])$.

The aim of the present paper is to establish an analog of Theorem A for the class of entire functions of improved regular growth with zeros on a finite system of rays. Our main result is the following theorem.

Theorem 1. An entire function $f$ of order $\rho \in(0,+\infty)$ with zeros on a finite system of rays $\left\{z: \arg z=\psi_{j}\right\}, j \in\{1, \ldots, m\}, 0 \leq \psi_{1}<\psi_{2}<\ldots<\psi_{m}<2 \pi$, is a function of improved regular growth if and only if for certain $\rho_{2} \in(0, \rho)$ and $k_{0} \in \mathbb{Z}$ and each $k \in\left\{k_{0}, k_{0}+1, \ldots, k_{0}+m-1\right\}$, one has

$$
c_{k}(r, F)=d_{k} r^{\rho}+o\left(r^{\rho_{2}}\right), \quad r \rightarrow+\infty, \quad r \notin \Omega, \quad d_{k} \in \mathbb{C} .
$$




\section{PRELIMINARIES}

In the proof of Theorem 1, we use the following auxiliary statements.

Lemma 1 ([7, 15]). An entire function $f$ of order $\rho \in(0,+\infty)$ with zeros on a finite system of rays $\left\{z: \arg z=\psi_{j}\right\}, j \in\{1, \ldots, m\}, 0 \leq \psi_{1}<\psi_{2}<\ldots<\psi_{m}<2 \pi$, is a function of improved regular growth if and only if for a certain $\rho_{3} \in(0, \rho)$ and each $j \in\{1, \ldots, m\}$

$$
n\left(t, \psi_{j} ; f\right)=\Delta_{j} t^{\rho}+o\left(t^{\rho_{3}}\right), \quad t \rightarrow+\infty, \quad \Delta_{j} \in[0,+\infty),
$$

and, in addition, for $\rho \in \mathbb{N}$ and certain $\rho_{4} \in(0, \rho)$ and $\delta_{f} \in \mathbb{C}$, one has

$$
\sum_{0<\left|\lambda_{n}\right| \leq r} \lambda_{n}^{-\rho}=\delta_{f}+o\left(r^{\rho_{4}-\rho}\right), \quad r \rightarrow+\infty .
$$

In this case,

$$
h(\varphi)=\sum_{j=1}^{m} h_{j}(\varphi), \quad \rho \in(0,+\infty) \backslash \mathbb{N},
$$

where $h_{j}(\varphi)$ is the $2 \pi$-periodic function defined on the interval $\left[\psi_{j}, \psi_{j}+2 \pi\right)$ by the equality $h_{j}(\varphi)=\frac{\pi \Delta_{j}}{\sin \pi \rho} \cos \rho\left(\varphi-\psi_{j}-\pi\right)$. In the case $\rho \in \mathbb{N}$, we have

$$
h(\varphi)=\left\{\begin{array}{l}
\tau_{f} \cos \left(\rho \varphi+\theta_{f}\right)+\sum_{j=1}^{m} h_{j}(\varphi), \quad \rho=p, \\
Q_{\rho} \cos \rho \varphi, \quad \rho=p+1,
\end{array}\right.
$$

where $\tau_{f}=\left|\delta_{f} / \rho+Q_{\rho}\right|, \theta_{f}=\arg \left(\delta_{f} / \rho+Q_{\rho}\right)$ and $h_{j}(\varphi)$ is the $2 \pi$-periodic function defined on the interval $\left[\psi_{j}, \psi_{j}+2 \pi\right)$ by the equality $h_{j}(\varphi)=\Delta_{j}\left(\pi-\varphi+\psi_{j}\right) \sin \rho\left(\varphi-\psi_{j}\right)-\frac{\Delta_{j}}{\rho} \cos \rho\left(\varphi-\psi_{j}\right)$.

Lemma 2 ([8]). Let $f$ be an entire function of order $\rho \in(0,+\infty)$ with zeros on a finite system of rays $\left\{z: \arg z=\psi_{j}\right\}, j \in\{1, \ldots, m\}, 0 \leq \psi_{1}<\psi_{2}<\ldots<\psi_{m}<2 \pi$. If $f$ is of improved regular growth, then for a certain $\rho_{5} \in(0, \rho)$ and each $k \in \mathbb{Z}$, one has

$$
c_{k}(r, \log |f|)=c_{k} r^{\rho}+o\left(r^{\rho_{5}}\right), \quad r \rightarrow+\infty,
$$

where

$$
c_{k}:=\frac{1}{2 \pi} \int_{0}^{2 \pi} e^{-i k \varphi} h(\varphi) d \varphi=\frac{\rho}{\rho^{2}-k^{2}} \sum_{j=1}^{m} \Delta_{j} e^{-i k \psi_{j}}, \quad \Delta_{j} \in[0,+\infty),
$$

if $\rho$ is a noninteger number, and

$$
c_{k}=\left\{\begin{array}{l}
\frac{\rho}{\rho^{2}-k^{2}} \sum_{j=1}^{m} \Delta_{j} e^{-i k \psi_{j}}, \quad|k| \neq \rho=p, \\
\frac{\tau_{f} e^{i \theta_{f}}}{2}-\frac{1}{4 \rho} \sum_{j=1}^{m} \Delta_{j} e^{-i \rho \psi_{j}}, \quad k=\rho=p, \\
0, \quad|k| \neq \rho=p+1, \\
\frac{Q_{\rho}}{2}, \quad k=\rho=p+1,
\end{array}\right.
$$

if $\rho \in \mathbb{N}$. Conversely, if for certain $\rho_{5} \in(0, \rho)$ and $k_{0} \in \mathbb{Z}$ and each $k \in\left\{k_{0}, k_{0}+1, \ldots, k_{0}+\right.$ $m-1\}$, relation (4) with $c_{k}$ defined by (5) and (6) be true, then $f$ is an entire function of improved regular growth. 


\section{Proof of Theorem 1}

Necessity. Let $f$ be an entire function of improved regular growth of order $\rho \in(0,+\infty)$ with zeros on a finite system of rays $\left\{z: \arg z=\psi_{j}\right\}, j \in\{1, \ldots, m\}, 0 \leq \psi_{1}<\psi_{2}<\ldots<$ $\psi_{m}<2 \pi$. Then, by Lemma 1 , for a certain $\rho_{3} \in(0, \rho)$ and each $j \in\{1, \ldots, m\}$ holds $(2)$ and, according to Lemma 2 , for a certain $\rho_{5} \in(0, \rho)$ and each $k \in \mathbb{Z}$, one has (4) with $c_{k}$ defined by (5) and (6). In view of this, since

$$
n_{k}(r, f)=\sum_{j=1}^{m} e^{-i k \psi_{j}} n\left(r, \psi_{j} ; f\right), \quad k \in \mathbb{Z},
$$

and ([13, p. 43])

$$
c_{k}(r, F)=n_{k}(r, f)+k^{2} \int_{0}^{r} \frac{c_{k}(t, \log |f|)}{t} d t+k c_{k}(r, \log |f|), \quad k \in \mathbb{Z}, \quad r \notin \Omega,
$$

then using (2), (4)-(6), for a certain $\rho_{2} \in(0, \rho)$ and each $k \in \mathbb{Z}$, we obtain

$$
c_{k}(r, F)=d_{k} r^{\rho}+o\left(r^{\rho_{2}}\right), \quad r \rightarrow+\infty, \quad r \notin \Omega,
$$

where

$$
d_{k}=\frac{\rho}{\rho-k} \sum_{j=1}^{m} \Delta_{j} e^{-i k \psi_{j}},
$$

if $\rho$ is a noninteger number, and (for $\rho=p+1$ equality (2) holds with $\Delta_{j}=0$, because $\left.\sum_{n \in \mathbb{N}}\left|\lambda_{n}\right|^{-p-1}<+\infty\right)$

$$
d_{k}=\left\{\begin{array}{l}
\frac{\rho}{\rho-k} \sum_{j=1}^{m} \Delta_{j} e^{-i k \psi_{j}}, \quad|k| \neq \rho=p, \\
\rho \tau_{f} e^{i \theta_{f}}+\frac{1}{2} \sum_{j=1}^{m} \Delta_{j} e^{-i \rho \psi_{j}}, \quad k=\rho=p, \\
0, \quad|k| \neq \rho=p+1, \\
\rho Q_{\rho}, \quad k=\rho=p+1,
\end{array}\right.
$$

if $\rho \in \mathbb{N}$. Thus, the relation (1) holds.

Sufficiency. Let equality (1) is true. Then, using (1) and the relation ([13, p. 43])

$$
n_{k}(r, f)=c_{k}(r, F)-k \int_{0}^{r} \frac{c_{k}(t, F)}{t} d t, \quad k \in \mathbb{Z}
$$

for certain $\rho_{2} \in(0, \rho)$ and $k_{0} \in \mathbb{Z}$ and each $k \in\left\{k_{0}, k_{0}+1, \ldots, k_{0}+m-1\right\}$, we obtain

$$
n_{k}(r, f)=d_{k} r^{\rho}-k \int_{0}^{r}\left(d_{k} t^{\rho-1}+o\left(t^{\rho_{2}-1}\right)\right) d t+o\left(r^{\rho_{2}}\right)=d_{k}(1-k / \rho) r^{\rho}+o\left(r^{\rho_{2}}\right),
$$

as $\Omega \not \supset r \rightarrow+\infty$, where $d_{k}$ are defined by (7) and (8). Further, without loss of generality, we can assume that $k_{0}=0$. Then, by analogy with [8, p. 1957] (see also [11, p. 127]), for $k \in\{0,1, \ldots, m-1\}$ we get

$$
n_{0}(r, f)=n\left(r, \psi_{1} ; f\right)+n\left(r, \psi_{2} ; f\right)+\ldots+n\left(r, \psi_{m} ; f\right) \text {, }
$$




$$
\begin{gathered}
n_{1}(r, f)=e^{-i \psi_{1}} n\left(r, \psi_{1} ; f\right)+e^{-i \psi_{2}} n\left(r, \psi_{2} ; f\right)+\ldots+e^{-i \psi_{m}} n\left(r, \psi_{m} ; f\right), \\
\ldots \ldots \ldots \ldots \ldots \ldots \ldots \ldots \ldots \ldots \ldots \ldots \ldots \ldots \ldots \ldots \ldots \ldots \ldots \ldots \ldots \ldots \ldots \ldots \ldots \ldots \ldots \ldots \ldots \ldots \ldots \ldots \ldots \ldots \\
n_{m-1}(r, f)=e^{-i(m-1) \psi_{1}} n\left(r, \psi_{1} ; f\right)+e^{-i(m-1) \psi_{2}} n\left(r, \psi_{2} ; f\right)+\ldots+e^{-i(m-1) \psi_{m}} n\left(r, \psi_{m} ; f\right) .
\end{gathered}
$$

This is a system of linear equations for the unknowns $n\left(r, \psi_{j} ; f\right), j \in\{1, \ldots, m\}$. Its determinant is the nonzero Vandermonde determinant:

$$
\left|\begin{array}{cccc}
1 & 1 & \ldots & 1 \\
e^{-i \psi_{1}} & e^{-i \psi_{2}} & \ldots & e^{-i \psi_{m}} \\
\ldots & \ldots & \ldots & \ldots \\
e^{-i(m-1) \psi_{1}} & e^{-i(m-1) \psi_{2}} & \ldots & e^{-i(m-1) \psi_{m}}
\end{array}\right| \neq 0 .
$$

Therefore, the functions $n\left(r, \psi_{j} ; f\right), j \in\{1, \ldots, m\}$, can be represented as linear combinations of the functions $n_{k}(r, f), k \in\{0,1, \ldots, m-1\}$. Solving this system by the Cramer rule and using (9), we obtain

$$
n\left(r, \psi_{j} ; f\right)=\Delta_{j} r^{\rho}+o\left(r^{\rho_{3}}\right), \quad r \rightarrow+\infty, \quad r \notin \Omega,
$$

for a certain $\rho_{3} \in(0, \rho)$ and each $j \in\{1, \ldots, m\}$. Since the functions $n\left(r, \psi_{j} ; f\right)$ are continuous on $[0,+\infty) \backslash \Omega$, we get relation (2). Let us now prove the equality (3). Since ([13, p. 43])

$$
c_{k}(r, F)=2 k c_{k}(r, \log |f|)+\sum_{\left|\lambda_{n}\right| \leq r}\left(\frac{\bar{\lambda}_{n}}{r}\right)^{k}, \quad k \in \mathbb{N},
$$

and for $k=\rho=p$ we have $([7$, p. 21])

$$
c_{\rho}(r, \log |f|)=\frac{1}{2} Q_{\rho} r^{\rho}+\frac{1}{2 \rho} \sum_{0<\left|\lambda_{n}\right| \leq r}\left(\left(\frac{r}{\lambda_{n}}\right)^{\rho}-\left(\frac{\bar{\lambda}_{n}}{r}\right)^{\rho}\right),
$$

then, using formulas $(1),(8)$ and the identity $\sum_{j=1}^{m} \Delta_{j} e^{-i \rho \psi_{j}}=0, \rho \in \mathbb{N}$, for a certain $\rho_{4} \in(0, \rho)$ we get

$$
\begin{aligned}
\sum_{0<\left|\lambda_{n}\right| \leq r} \lambda_{n}^{-\rho} & =r^{-\rho} c_{\rho}(r, F)-\rho Q_{\rho}=d_{\rho}-\rho Q_{\rho}+o\left(r^{\rho_{2}-\rho}\right) \\
& =\rho\left(\tau_{f} e^{i \theta_{f}}-Q_{\rho}\right)+o\left(r^{\rho_{4}-\rho}\right)=\delta_{f}+o\left(r^{\rho_{4}-\rho}\right), \quad r \rightarrow+\infty
\end{aligned}
$$

Hence, equality (3) holds for $\rho=p$. In the case $\rho=p+1$, condition (3) follows from (2) (see [7, Remark 2, p. 23]). Thus, according to Lemma 1, the entire function $f$ is a function of improved regular growth. This completes the proof of Theorem 1.

\section{REFERENCES}

[1] Gol'dberg A.A. B.Ya. Levin is a creator of the theory of entire functions of completely regular growth. Mat. Fiz., Anal., Geom. 1994, 1 (2), 186-192. (in Russian) 
[2] Gol'dberg A.A., Korenkov N.E. Asymptotic behavior of logarithmic derivative of entire function of completely regular growth. Sib. Math. J. 1980, 21 (3), 363-375. doi:10.1007/BF00968180 (translation of Sib. Mat. Zh. 1980, 21 (3), 63-79. (in Russian))

[3] Gol'dberg A.A., Levin B.Ya., Ostrovskii I.V. Entire and meromorphic functions. In: VINITI Series in Contemporary Problems of Mathematics, Fundamental Trends, 85. VINITI, Moscow 1991, pp. 5-186. (in Russian)

[4] Gol'dberg A.A., Sodin M.L., Strochik N.N. Meromorphic functions of completely regular growth and their logarithmic derivatives. Sib. Math. J. 1992, 33 (1), 34-40. doi:10.1007/BF00972934 (translation of Sib. Mat. Zh. 1992, 33 (1), 44-52. (in Russian))

[5] Gol'dberg A.A., Strochik N.N. Asymptotic behavior of meromorphic functions of completely regular growth and of their logarithmic derivatives. Sib. Math. J. 1985, 26 (6), 802-809. doi:10.1007/BF00969100 (translation of Sib. Mat. Zh. 1985, 26 (6), 29-38. (in Russian))

[6] Hirnyk M.O. Subharmonic functions of improved regular growth. Dopov. Nats. Akad. Nauk Ukr. 2009, 4, 13-18. doi:10.1007/s11253-012-0624-2 (in Ukrainian)

[7] Khats' R.V. On entire functions of improved regular growth of integer order with zeros on a finite system of rays. Mat. Stud. 2006, 26 (1), 17-24.

[8] Khats' R.V. Regularity of growth of Fourier coefficients of entire functions of improved regular growth. Ukr. Math. J. 2012, 63 (12), 1953-1960. doi:10.1007/s11253-012-0624-2 (translation of Ukr. Mat. Zh. 2011, 63 (12), 1717-1723. (in Ukrainian))

[9] Khats' R.V. Asymptotic behavior of averaging of entire functions of improved regular growth. Carpathian Math. Publ. 2013, 5 (1), 129-133. doi:10.15330/cmp.5.1.129-133

[10] Khats' R.V. Asymptotic behavior of entire functions of improved regular growth in the metric of $L^{p}[0,2 \pi]$. Carpathian Math. Publ. 2013, 5 (2), 341-344. doi:10.15330/cmp.5.2.341-344

[11] Kondratyuk A.A. Fourier series and meromorphic functions. Vyshcha shkola, Lviv, 1988. (in Russian)

[12] Levin B.Ya. Distribution of zeros of entire functions. In: Transl. Math. Monogr., 5, Amer. Math. Soc., Providence, R.I., 1980.

[13] Vasylkiv Ya.V. Asymptotic behavior of logarithmic derivatives and logarithms of meromorphic functions of completely regular growth in the metric of $L^{p}[0,2 \pi]$. I. Mat. Stud. 1999, 12 (1), 37-58. (in Ukrainian)

[14] Vynnyts'kyi B.V., Khats' R.V. On asymptotic behavior of entire functions of noninteger order. Mat. Stud. 2004, 21 (2), 140-150. (in Ukrainian)

[15] Vynnyts'kyi B.V., Khats' R.V. On growth regularity of entire function of noninteger order with zeros on a finite system of rays. Mat. Stud. 2005, 24 (1), 31-38. (in Ukrainian)

Received 25.01.2019

Хаць Р.В. Регулярне зростання коефічієнтів Фур’є логарифмічної похідної иілих функиій покращеного регулярного зростання // Буковинський матем. журнал - 2019. - Т.7, №1. - C. 114-120.

Нехай $f$ - ціла функція, $f(0)=1,\left(\lambda_{n}\right)$ - послідовність її нулів, $\Omega=\left\{\left|\lambda_{n}\right|: n \in\right.$ $\mathbb{N}\}$ i $F(z)=z f^{\prime}(z) / f(z), z=r e^{i \varphi}$. Ціла функція $f$ називається функцією покращеного регулярного зростання, якщо для деяких $\rho \in(0,+\infty), \rho_{1} \in(0, \rho)$ і $2 \pi$-періодичної $\rho$ тригонометрично опуклої функції $h(\varphi) \not \equiv-\infty$ існує множина $U \subset \mathbb{C}$, яка міститься в 
об'єднанні кругів із скінченною сумою радіусів така, що $\log |f(z)|=|z|^{\rho} h(\varphi)+o\left(|z|^{\rho_{1}}\right), U \not \supset$ $z=r e^{i \varphi} \rightarrow \infty$. В роботі доведено, що для того щоб ціла функція $f$ порядку $\rho \in(0,+\infty)$ 3 нулями на скінченній системі променів $\left\{z: \arg z=\psi_{j}\right\}, j \in\{1, \ldots, m\}, 0 \leq \psi_{1}<\psi_{2}<$ $\ldots<\psi_{m}<2 \pi$, була функцією покращеного регулярного зростання, необхідно і достатньо, щоб для деяких $\rho_{2} \in(0, \rho), k_{0} \in \mathbb{Z}$ i кожного $k \in\left\{k_{0}, k_{0}+1, \ldots, k_{0}+m-1\right\}$, виконувалось

$$
c_{k}(r, F)=\frac{1}{2 \pi} \int_{0}^{2 \pi} e^{-i k \varphi} F\left(r e^{i \varphi}\right) d \varphi=d_{k} r^{\rho}+o\left(r^{\rho_{2}}\right), \quad r \rightarrow+\infty, \quad r \notin \Omega, \quad d_{k} \in \mathbb{C} .
$$

Це доповнює результати А. Гольдберга, М. Содіна, М. Строчика, М. Коренкова та Я. Васильківа про функції цілком регулярного зростання. 